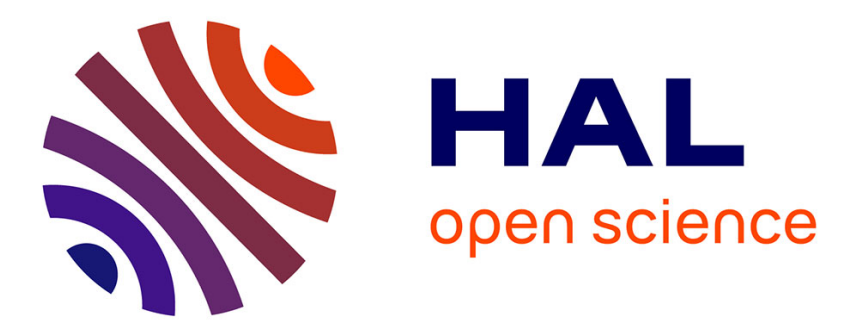

\title{
UAV-assisted supporting services connectivity in urban VANETs
}

\author{
Omar Sami Oubbati, Noureddine Chaib, Abderrahmane Lakas, Pascal \\ Lorenz, Abderrezak Rachedi
}

\section{- To cite this version:}

Omar Sami Oubbati, Noureddine Chaib, Abderrahmane Lakas, Pascal Lorenz, Abderrezak Rachedi. UAV-assisted supporting services connectivity in urban VANETs. IEEE Transactions on Vehicular Technology, 2019, 68 (4), pp.3944-3951. 10.1109/TVT.2019.2898477 . hal-02334367

\section{HAL Id: hal-02334367 https://hal.science/hal-02334367}

Submitted on 2 Nov 2019

HAL is a multi-disciplinary open access archive for the deposit and dissemination of scientific research documents, whether they are published or not. The documents may come from teaching and research institutions in France or abroad, or from public or private research centers.
L'archive ouverte pluridisciplinaire HAL, est destinée au dépôt et à la diffusion de documents scientifiques de niveau recherche, publiés ou non, émanant des établissements d'enseignement et de recherche français ou étrangers, des laboratoires publics ou privés. 


\title{
UAV-assisted supporting services connectivity in urban VANETs
}

\author{
Omar Sami Oubbati, Member, IEEE, Noureddine Chaib, Senior Member, IEEE, \\ Abderrahmane Lakas, Member, IEEE, Pascal Lorenz, Senior Member, IEEE, \\ Abderrezak Rachedi, Senior Member, IEEE
}

\begin{abstract}
To keep the services and applications of Intelligent Transportation System (ITS) stable and active, Vehicular Ad hoc Networks (VANETs) are considered as an essential building block to maintain and manage its features. A wide deployment of VANETs is possible only after addressing numerous research challenges. One of the most complicated issues consists in designing a routing strategy, taking into consideration several serious constraints, and especially in a network such as VANET. The severity of these issues would be increased significantly when a VANET is deployed over an urban area, where we distinguish the high mobility of nodes and existing obstructions (e.g., buildings, bridges, tunnels, etc.). In this paper, an efficient routing solution based on a flooding technique is conceived to make the data delivery more reliable and to guarantee robust paths. Vehicles can cooperate in ad hoc fashion with existing Unmanned Aerial Vehicles (UAVs). This kind of collaboration provides reliable routing paths and ensures alternative solutions in the case of path failures. Furthermore, a prediction technique is used to expect the expiration time of each discovered path. To limit the overhead over the network, all control packets are characterized by their static size making the originality of this work. Based on the simulation outputs, we discuss the performances of the proposed approach as compared with other dedicated previous schemes in terms of several metrics. The obtained results demonstrate that the hybrid communication between vehicles and UAVs based on the proposed flooding technique is perfectly suited to improve the data delivery process.
\end{abstract}

Index Terms-Routing protocols; VANETs; UAVs; Connectivity; Simulation; Flooding process.

\section{INTRODUCTION}

Vehicular Ad hoc Networks (VANETs) have paved the path to numerous road-safety applications where Internet access is considered as their indisputable support [1]. Internet access is generally supplied by fixed Road Side Units (RSUs) along the roads acting as gateways and it used by vehicles to share critical information about the road (e.g., crashes) with security services [2]. However, the existing obstructions and the high mobility of vehicles can disturb the communication links between vehicles and RSUs resulting in many disconnections.

Copyright (C) 2019 IEEE. Personal use of this material is permitted. However, permission to use this material for any other purposes must be obtained from the IEEE by sending a request to pubs-permissions@ieee.org.

O.S. Oubbati and N. Chaib are with the Computer Science and mathematics Laboratory, University Amar Telidji of Laghouat, BP 37G, Ghardaïa Road, 03000 Laghouat, Algeria. E-mail: \{s.oubbati,n.chaib\} @lagh-univ.dz.

A. Lakas is with College of Information Technology, United Arab Emirates University, United Arab Emirates. E-mail: alakas@uaeu.ac.ae

P. Lorenz is with University of Haute Alsace, France. E-mail: pascal.lorenz@uha.fr

A. Rachedi is with Paris-EST University (UPEM), France. E-mail: rachedi@u-pem.fr
Recently in modern cities, Unmanned Aerial Vehicles (UAVs) or what is referred to as drones have seen their numbers significantly increase. UAVs can be considered as the suitable candidates to improve the connectedness of VANETs [3]. Moreover, UAVs can avoid existing obstacles and participate reliably in the data delivery by cooperating with existing VANETs. Based on this concept, only a limited number of routing protocols have been proposed in the literature suffering from several drawbacks. In [4], UAVs in the sky form an aerial subnetwork to assist existing VANETs on the ground. Due to their flexible mobility, the UAVs act as intermediate relays when a disconnection in VANETs occurs. This protocol requires a fully connected network and it cannot tolerate a few seconds of network fragmentation. This is not reasonable since, as widely known, VANETs are characterized by their high mobility.

To overcome the aforementioned issues, the protocol proposed in [5] tries to gradually build routing paths, road segment by road segment at each intersection by combining three parameters: (i) the connectivity, (ii) the traffic density, and (iii) the distance between the communicating nodes. However, this protocol has many drawbacks such as the neglect of the real distribution of vehicles in the routing paths, UAVs are involved as relays only in the case of disconnections, and the obstacles are overlooked causing the signals' disruption.

The work presented in [6] has addressed some drawbacks of [5]. Indeed, UAVs are exploited to calculate the real distribution and the connectivity degree of vehicles more accurately based on the intercepted Hello packets exchanged by vehicles while overcoming the present obstacles. Nevertheless, the UAVs are only used when no routing path among vehicles is found on the ground. Thus, the UAVs are not fully exploited.

A novel technique is adopted in [7]. A discovery process is initiated by flooding a route request (RREQ) on-demand between a pair of communicating nodes. Once the routing decision is made by the destination, a route reply (RREP) packet including all the discovered paths is sent back through the primarily selected path. The source, in turn, sends the data packet including in its header the same information as in the RREP packet in order to be used later during the maintenance process. As a drawback, the exchanged control packets, as well as the data packet, have a dynamic size, which increases significantly the overhead when there is an important number of discovered paths.

To recapitulate, different solutions of the aforementioned drawbacks are proposed and are summarized in TABLE [ 
TABLE I: Drawbacks and different proposed solutions of UAV-assisted VANET protocols.

\begin{tabular}{|c|c|c|}
\hline Protocols & Drawbacks & Possible solutions \\
\hline Ref. 4 & $\begin{array}{l}\text { - Permanent connectedness of the network. } \\
\text { - Ignorance of existing obstructions. }\end{array}$ & $\begin{array}{l}\text { - Consider the high mobility and network fragmentations. } \\
\text { - Obstructions awareness by fully exploiting UAVs in the sky. }\end{array}$ \\
\hline Ref. |5| & $\begin{array}{l}\text { - Unawareness of vehicles' real dispersion on road segments. } \\
\text { - Slow maintenance process and bad effects of existing obstructions. }\end{array}$ & $\begin{array}{l}\text { - Calculate the real distribution of vehicles on discovered routing paths. } \\
\text { - Find reliable alternative solutions in the sky and avoid carrying packets. }\end{array}$ \\
\hline Ref. |6] & $\begin{array}{l}\text { - UAVs not fully exploited. } \\
\text { - Inefficient maintenance process. }\end{array}$ & $\begin{array}{l}\text { - Consider UAVs for both relaying packets and obstruction avoidance. } \\
\text { - Provide several alternative solutions both in the sky and on the ground. }\end{array}$ \\
\hline Ref. [7] & $\begin{array}{l}\text { - Dynamic size of control packets. } \\
\text { - Unreachable alternative solutions in the maintenance process. }\end{array}$ & $\begin{array}{l}\text { - Use static size control packets to limit the overhead. } \\
\text { - Envisage several reachable alternative solutions to get the destination. }\end{array}$ \\
\hline
\end{tabular}

The main objective of this research is to take into account the different solutions proposed in TABLE I at once and introduce a new routing concept. The contributions of this paper are as follows:

- An extension of our earlier work [7] is proposed to find the most appropriate routing path while overcoming the aforementioned issues. Indeed, the current work considers: (i) the route discovery based on fixed size control packets that are used intelligently to limit the overhead while avoiding obstructions by transmitting them through UAVs in the sky, (ii) the routing decision to select the adequate path for the data delivery, (iii) the data delivery to transmit the data packet through the selected path, and (iv) the maintenance strategy to recover path failures.

- Several experiments have studied the effects of having UAVs as members belonging to discovered routing paths.

The rest of this paper is organized as follows. In Section III. we review a set of related works that are relevant to our work. After that, we describe the main functionalities of the proposed work in Section III. In Section IV] we detail the simulation results and their analysis. Finally, we conclude the paper in Section $\mathrm{V}$.

\section{RELATED WORK}

Routing protocols based on the flooding process are preferable for VANETs [1]. The scheme proposed in [8] exploits the flooding to extract the velocity vectors of vehicles in order to classify them into different groups. Then, the destination selects the most stable path for the data delivery. As a drawback, the extra overhead caused by the dynamic size of control packets and the neglect of the distributions of vehicles.

Recently, UAVs have been often exploited to relay messages for ground terminals. In [9], vehicle-assist resilient information and network system are designed both to overcome the failure of communication infrastructures in the disaster area. This system is composed of three major components: (i) smartphone apps providing a multitude of services, (ii) mobile stations or UAVs to assist the communication between smartphone and servers, and (iii) servers collecting, analyzing, and making decisions based on the provided user data. The authors in [10] study the automatic ground map building and efficient path planning in unmanned aerial/ground vehicles (UAV/UGV) cooperative systems. UAVs provide an aerial vision which is then processed, corrected, and the different obstacles are automatically recognized. A communication over multiple UAVs is considered in [11]. This technique allocates the center coordinates of UAVs and the radius of their trajectory in order to improve the delay performance of UAV swarm networks. In [12], UAVs are used as relays to enhance the communication performance of VANETs and to resist against jamming due to the high mobility, altitude, and Line of Sight (LoS).

The deployment of UAV-to-Ground architecture sometimes requires the knowledge of routing techniques adopted within UAVs. When a network of UAVs suffers from disconnections, the scheme proposed in [13] uses a data delivery technique based essentially on the Greedy Geographic Forwarding (GGF). It employs location information of UAVs to select the adequate intermediate UAV. In [14], the intermediate UAVs can estimate the current position of the source UAV, and thus providing the transition distance of the packet. This can be used as a metric during the routing decision. Its main drawback is that the important number of extra packets used in the data delivery can cause the problem of congestion.

In this work, a new improved flooding process is introduced to establish routing paths with the minimum of overhead. For this purpose, a couple of techniques are included in order to minimize the re-initialization of the discovery phase when a disconnection in VANET occurs. These techniques are used during the discovery phase such as the prediction technique to define the expiration time of each discovered path and also during the maintenance phase when there is a path failure.

\section{UAV-ASSISTED SUPPORTING SERVICES CONNECTIVITY IN URBAN VANETS}

In this section, we propose a new reactive-based routing protocol using cooperating UAVs to ensure a durable connectivity even when the network on the ground (VANET) is sparsely connected. As shown in Fig. 1, the key idea behind the protocol is to exploit the route discovery to have accurate details about the connectivity degree of the routing paths between the communicating nodes. The established paths do not only consist of vehicles, but also they can be made of UAVs or both. A routing decision has to be made by the destination based on many criteria such as the traffic density, the expiration time of the path, and the real distribution of nodes constituting the path. Once a path is selected, data packets are delivered through the selected path using the greedy forwarding technique. When the selected path disconnects due to the dynamic nature of such networks, an efficient maintenance strategy tries to find alternative solutions which can be provided in the majority of cases by UAVs flying over the area. Before describing each element of our protocol, we first describe the system model of this work. 


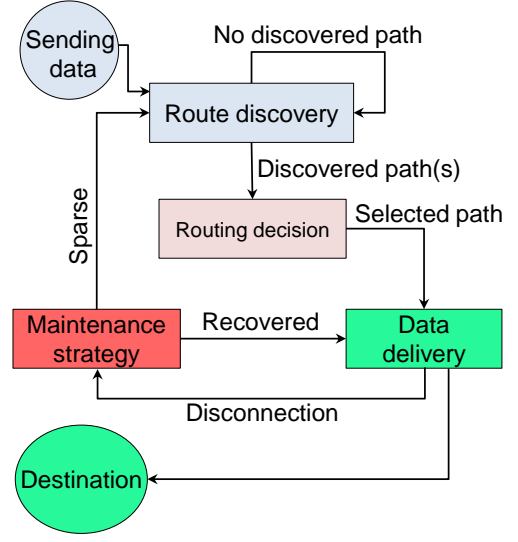

Fig. 1: Functioning of our protocol.

\section{A. System model}

In this work, the network (i.e., each road segment and the airspace) is supposed to be divided into identified fixed zones (c.f., Fig. 5p. The size of each ground zone depends on the communication range of vehicles $(\approx 300 \mathrm{~m})$. As for the aerial zones, we study two different cases: (i) small aerial zones and (ii) large aerial zones according to the communication range of UAVs, which is $\approx 300 \mathrm{~m}$ or $\approx 1000 \mathrm{~m}$, respectively. In the first case, the reason behind the choice of the same coverage as with vehicles is to avoid both the degradation of the spectral efficiency and much larger interference during the communications. Secondly, as demonstrated and adopted in [1], [15], a large coverage provides a high LoS probability in order to support more vehicles. We note that we consider UAVs in the form of small Quad-Copters, which can fly at low and constant altitudes (i.e., do not exceed $\approx 300 \mathrm{~m}$ during the flight) [16]. This restriction is imposed in order to avoid restricted flight zones reserved for other aircraft [17]. Each mobile node (Vehicle/UAV) in the network is equipped with a global positioning system (GPS) and a digital map to obtain its current geographical position and that of each fixed zone, respectively. In addition, all nodes maintain and update their own routing tables. Also, it is supposed that there is no energy restriction since each node is equipped with rechargeable batteries, which can be continuously recharged by the nodes' resources such as solar energy, gasoline, and electrical energy [3]. Vehicles and UAVs are equitably distributed and exchanging data packets over the network using integrated wireless interfaces using IEEE 802.11p at the MAC layer, which is the most suitable for vehicular communications [18]. In our case, five possible kinds of wireless communications, which can be carried out successfully if the communicating nodes are within LoS communication range of each other (c.f., Fig. 2). Vehicle-to-Vehicle (V2V) is not possible when there is an obstacle between the communicating vehicles. Vehicleto-UAV (V2U) and UAV-to-UAV (U2U) are always possible if the communicating nodes are within the transmission range of each other. Vehicle-to-Road-Side-Unit (V2R) and UAV-to-Road-Side-Unit (U2R) are established between RSUs and existing vehicles only when certain applications need to be run such as Internet access.

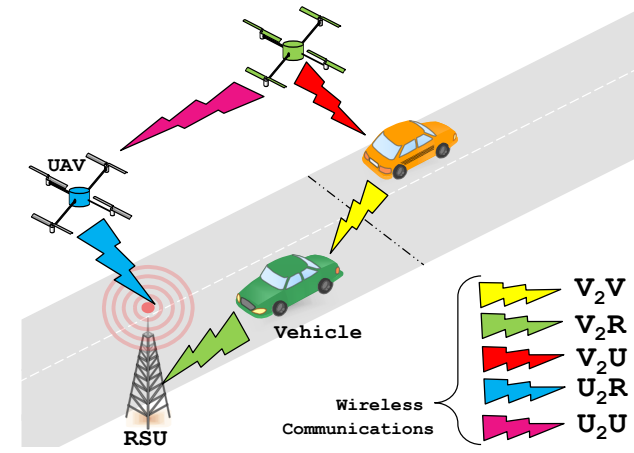

Fig. 2: Existing kinds of wireless communication.

The authors in [19], [20] have demonstrated that static ground base stations (BSs) provide performance enhancement in terms of delay over the mobility of UAVs, and especially when strict delay is considered. However, UAVs are cheaper compared with ground BSs and they are easily deployable in a three dimensional (3D) to act as relays enhancing the ground network connectivity. Moreover, the performance gain will be higher if the mobility of UAVs will be dynamic and adjustable and the data is delay tolerant. TABLE[I] presents a brief comparison between UAVs and terrestrial BSs.

TABLE II: UAVs vs. BSs.

\begin{tabular}{|l|l|l|}
\cline { 2 - 3 } \multicolumn{1}{c|}{} & UAVs & BSs \\
\hline Price & Cheaper & Expensive \\
Deployment & 3D & 2D \\
Exploitation & Short-term & Long-term \\
Position & Unrestricted & Restricted \\
Movement & Dynamic & Static \\
LoS & High & Medium \\
Coverage & Large & Medium (in urban area) \\
\hline
\end{tabular}

In our work, random movements are adopted for all UAVs. Even if it is not quite suitable for FANETs [16], the reason behind adopting such kind of mobility model is to study the critical impact of random movements on the proposed routing protocol. Moreover, UAVs are fully exploited both in the discovered routing paths and as alternatives when the network is highly fragmented on the ground. This helps to decrease considerably the packet losses and delivery delay since the problem of obstacles no longer arises in the sky.

\section{B. Packet format}

The RREQ packet format is depicted in Fig. 3(a) The Flooding ID defines the route discovery to which the RREQ packet belongs. The Delay represents the time needed for a data delivery. When the Life time is expired, the packet is dropped. The Source and Destination identifiers and positions. The Movement information is modified at each hop as the RREQ packet crossing the network, where intermediate nodes add the required information about their movements (i.e., velocity, speed, and position). Each receiving node exploits the Movement information field with its own one to calculate the lifetime of the link. This value is then used to modify the $P E$ field if, and only if, the value of the 


\begin{tabular}{|c|c|c|c|}
\hline \multicolumn{4}{|c|}{16} \\
\hline \multicolumn{4}{|c|}{ Flooding ID } \\
\hline & lay & Life & time \\
\hline \multicolumn{4}{|c|}{ Source } \\
\hline \multicolumn{4}{|c|}{ Destination } \\
\hline \multicolumn{4}{|c|}{ Movement information } \\
\hline Zon & count & $\mathrm{N}(\mathrm{Z})$ & $\sigma$ \\
\hline $\mathrm{PE}(\mathrm{s})$ & DisZone & & $5 \mathrm{~N}$ \\
\hline
\end{tabular}

(a) A RREQ packet Format.

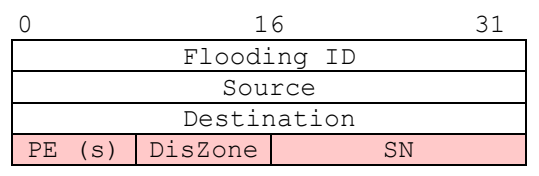

(b) A RREP packet Format.

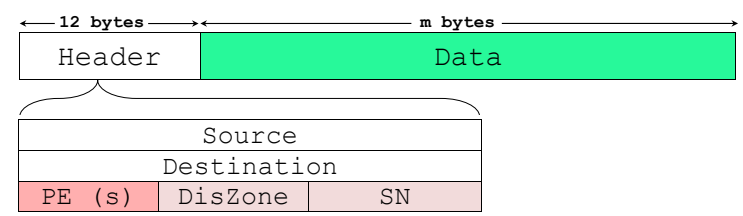

(c) Data packet Format.

Fig. 3: Format of routing packets.

already included $P E$ in the RREQ packet is higher than the calculated lifetime of the new wireless link. At the end of the flooding, $P E$ represents the minimum lifetime on a specified wireless link between two successive nodes belonging to this discovered path. The DisZone represents the weakest connected zone (i.e., the zone with the weakest wireless link in the discovered path). The $S N$ corresponds to a unique path, which exists between the source and target destination. The number of transited zones Zone count, the total number of nodes in the path $N(Z)$, and the real distribution of nodes in the path $\sigma$, are calculated progressively during the route discovery.

Once the destination has made the routing decision, it generates an RREP packet as depicted in Fig. 3(b). Copying the Flooding $I D$, the $P E$, the $S N$ and DisZone of the selected path, and the Source and Destination identifiers same as the received RREQ packet onto this new packet. At the reception of the RREP packet, the source node adds a new routing entry of the selected path. This will help to start the data transmission and maybe to send other data packets in the future if the selected path is not expired. The source generates a data packet with a header which does not exceed 12 bytes containing the same fields as in the RREP packet (c.f., Fig. 3(c) . The Source and Destination are used for the greedy forwarding technique. $S N$ denotes the selected path, which has to be transited during the data delivery. $P E$ and DisZone fields are checked before each data transmission by the source and intermediate nodes in order to determine whether this selected path is still valid to make other data transmissions or to find other alternative solutions in the case of a disconnection.

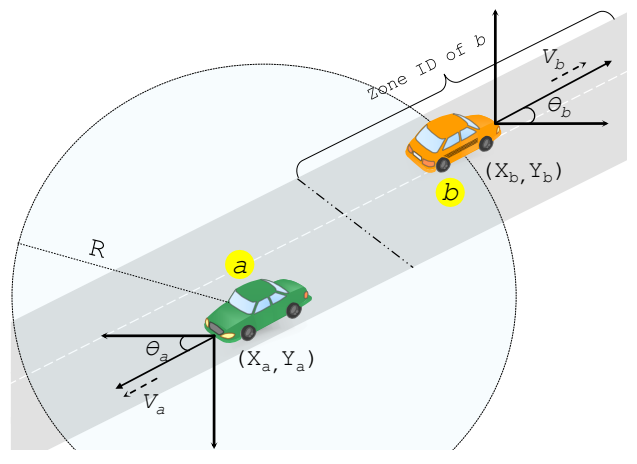

(a) Same altitudes.

\section{Calculation of the path expiration time PE}

$P E$ is the minimum remaining time of two nodes to stay connected in a given path. Two different scenarios are distinguished (c.f., Fig. 4): (i) two nodes with the same altitudes (e.g., two vehicles) and (ii) nodes with different altitudes (e.g., vehicles and UAVs). In the first example of Fig. 4(a), let $a$ and $b$ be two nodes with nonzero speeds $V_{a}$ and $V_{b}, R$ the Line-ofSight (LoS) transmission range of the two nodes, $\left(X_{a}, Y_{a}\right)$ and $\left(X_{b}, Y_{b}\right)$ their corresponding coordinates, and $\theta_{a}$ and $\theta_{b}$ their velocity angles. The lifetime of the link between the nodes $a$ and $b$ is calculated by using the same method in [21]:

$$
P E=\frac{-(i j+k m)+\sqrt{\left(i^{2}+k^{2}\right) R^{2}-(i m-j k)^{2}}}{i^{2}+k^{2}}
$$

where,

$$
\begin{aligned}
i & =V_{a} \cos \theta_{a}-V_{b} \cos \theta_{b} \\
k & =V_{a} \sin \theta_{a}-V_{b} \sin \theta_{b} \\
j & =X_{a}-X_{b} \\
m & =Y_{a}-Y_{b}
\end{aligned}
$$

In the second scenario (c.f., Fig. 4(b), we adopt the same method used in [22]. The link expiration time between two nodes $a$ and $b$ with a LoS range R, non zero speeds $V_{a}$ and $V_{b}$, their initial locations be $\left(X_{a}, Y_{a}, Z_{a}\right)$ and $\left(X_{b}, Y_{b}, Z_{b}\right)$, and their respective velocity angles $\theta_{a}, \phi_{a}$ and $\theta_{b}, \phi_{b}$. The link expiration time can be calculated as follows:

$$
P E=\frac{-n \pm \sqrt{n^{2}-4 m o}}{2 m}
$$

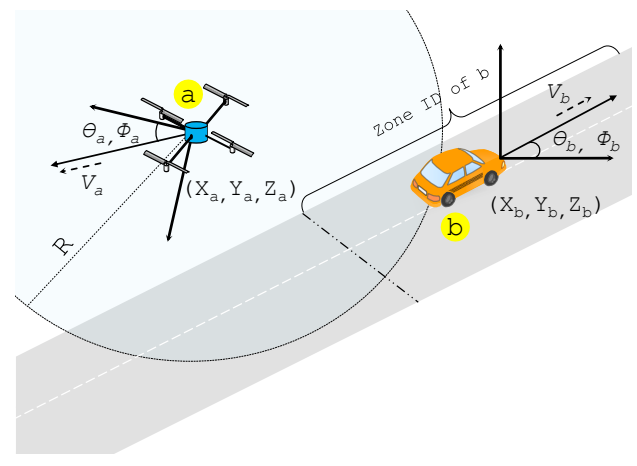

(b) Different altitudes.

Fig. 4: Path expiration time calculation. 
where,

$$
\begin{aligned}
m & =w^{2}+z^{2}+g^{2} \\
n & =2 i w+2 j z+2 k g \\
o & =i^{2}+j^{2}+k^{2}-R^{2} \\
i & =\left(X_{a}-X_{b}\right) \\
j & =\left(Y_{a}-Y_{b}\right) \\
k & =\left(Z_{a}-Z_{b}\right) \\
w & =\left(V_{a} \sin \theta_{a} \cos \phi_{a}-V_{b} \sin \theta_{b} \cos \phi_{b}\right) \\
z & =\left(V_{a} \sin \theta_{a} \sin \phi_{a}-V_{b} \sin \theta_{b} \sin \phi_{b}\right) \\
g & =\left(V_{a} \cos \theta_{a}-V_{b} \cos \theta_{b}\right)
\end{aligned}
$$

\section{Our approach functioning}

To illustrate our protocol functioning, let us consider the network shown in Fig. 5

1) Route discovery: The requesting vehicle $v_{1}$ located in the zone $A$ broadcasts an RREQ packet across the network to find all possible routing paths (i.e., zones' succession) towards the fixed destination gateway $y_{5}$ located in the zone $Z$. The flooding process is assigned with a unique ID and a sequence number $(S N)$ in the broadcasted RREQs, which helps to reduce the broadcast storm effect. For instance, if a received RREQ has the same SN and Flooding ID with a previously received one, or it comes from a zone where there is already a routing entry towards it (i.e., an RREQ cannot transit a reverse path of an already discovered path), it is automatically dropped. Otherwise, all necessary information included in the RREQ is cached in the current node's routing table. It should be stressed that the full paths are not stored, neither in the control packets (RREQs and RREPs) nor in the exchanged data packets due to their static size. All these criteria make the originality of our flooding process compared with traditional flooding techniques used by other protocols.

\begin{tabular}{|c|c|c|c|c|}
\hline \multicolumn{5}{|c|}{ Routing Table (Zone A) } \\
\hline Prev. Zone & Next Zone & Dest. ID/Zone & Flooding ID & Flooding SN \\
\hline- & B & 5 & 01 & B \\
\hline- & L & 5 & 01 & L \\
\hline$\cdots$ & $\cdots$ & $\cdots$ & $\cdots$ & $\cdots$ \\
\hline
\end{tabular}

Fig. 6: Routing table of $v_{1}$.

Therefore, each path is identified by a unique $S N$ (e.g., based on the next zones B and L, two different $S N s$ are defined: $S N=B$ and $S N=L$ ). For instance, in Fig. 6, two routing entries with the same ID representing the two possible paths are added to the routing table of $v_{1}$ ). $S N$ along with other information are updated in the RREQ which will be then re-broadcasted. It is worth noting that, the same routing table is maintained by vehicles at each zone. Moreover, at each intersection or a UAV relay, a new routing entry is added to the routing tables of the current forwarders (i.e., a new $S N$ with the same Flooding ID representing the novel path). The paths are built gradually, and at the same time, several parameters are progressively calculated using [23] for each path, such as the traffic density $N(Z)$, the number of nodes $N\left(Z_{i}\right)$ in each zone $Z_{i}$, the real distribution of nodes $\sigma$, the path expiration time $P E$, the number of transited zones Zone count, the average number of nodes $\mu$, and the delivery delay Delay.

2) Routing decision: After the reception of the RREQs, a set of updates has to be done in the routing table of gateway

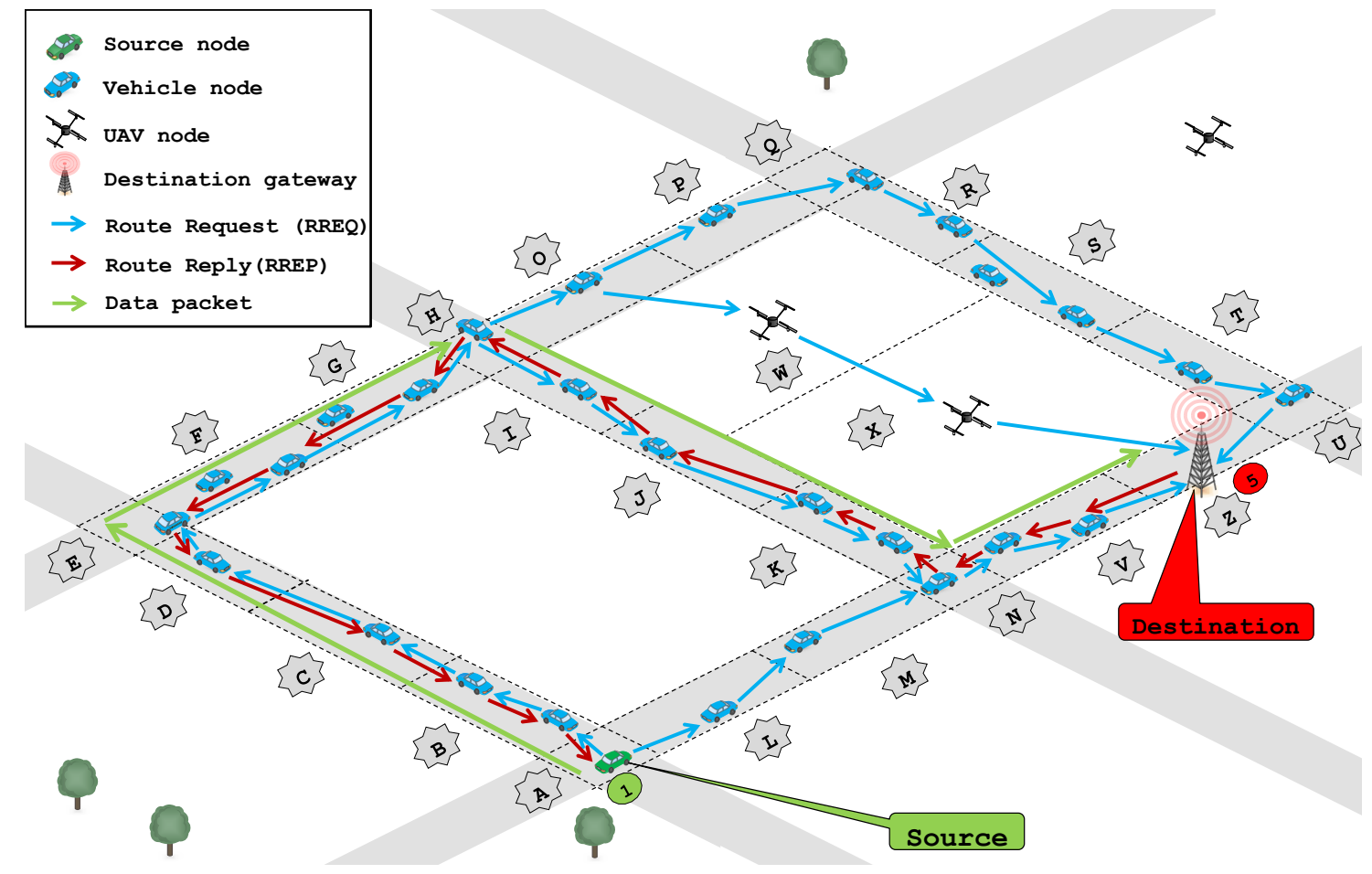

Fig. 5: Principle of functioning of our approach. 
(c.f., Fig. 7).

\begin{tabular}{|c|c|c|c|c|}
\hline \multicolumn{5}{|c|}{ Routing Table (Zone Z) } \\
\hline Prev. Zone & Next Zone & Dest. ID/Zone & Flooding ID & Flooding SN \\
\hline V & V & A & 01 & B \\
\hline V & V & A & 01 & L \\
\hline U & V & A & 01 & O \\
\hline X & V & A & 01 & W \\
\hline$\ldots$ & $\ldots$ & $\ldots$ & $\ldots$ & $\ldots$ \\
\hline
\end{tabular}

Fig. 7: Routing table of gateway 5 .

A score based on the already calculated parameters is calculated for each path as follows:

$$
\text { Score }=\left\lfloor\frac{P E}{\text { Delay }}\right\rfloor \times\left(\frac{N(Z)}{1+\sigma}\right)
$$

Where,

$$
\begin{array}{r}
N(Z)=\sum_{i=1}^{\text {Zone count }} N\left(Z_{i}\right) \\
\sigma=\sqrt{\frac{1}{\text { Zone count }} \times \sum_{i=1}^{\text {Zone count }}\left(N\left(Z_{i}\right)-\mu\right)^{2}}
\end{array}
$$

From the equation (1), we can notice the following remarks:

- The calculated Score has a proportional relationship with $N(Z)$ and $P E$ which play a key role to determine the connectivity degree of a path.

- The floor of $\frac{P E}{\text { Delay }}$ represents whether the corresponding path still remains connected or not during a data transmission. When $\left\lfloor\frac{P E}{\text { Delay }}\right\rfloor=0$, it means that there is a high probability of a disconnection during the data delivery. However, if $\left\lfloor\frac{P E}{\text { Delay }}\right\rfloor>0$, it means that we have a certain guarantee that this path remains connected during the data transmission.

Despite the high mobility of nodes, paths with high scores are suitable. This is because they can ensure a reliable data delivery since they have a high value of the path expiration time and an important number of relays ensuring more data packets to send.

gateway $_{5}$ selects the best path with the highest score, and updates the next zone in the routing table (i.e., the routing entry highlighted in red in Fig. 7p. The selected $S N$ (i.e., selected path), $P E$, DisZone, and the identifiers of the source and destination are included in the RREP, and sent back to the source through the selected path, which can be used later during the maintenance. At each zone transited by the RREP packet, a modification has to be done in the routing tables of vehicles located in these zones to indicate the selected next zone.

3) Data delivery: To have a deep understanding of the data delivery phase, the data packets are delivered through the succession of zones forming the selected routing path based on the greedy forwarding technique when it is possible. This helps to minimize the number of hops since the data packet is delivered to the closest node to the destination, and consequently, minimizing the delay of delivering. Moreover, this decomposition into zones is crucial for the knowledge of the exact location of link breakages when they occur. In addition, it can also help to find alternative solutions to avoid sparsely connected zones towards the target destination.

As shown in Fig. 5, at the reception of the RREP packet, the source vehicle starts the data delivery. For instance, when a vehicle located in the zone $C$ receives the data packet, it checks its header and compares all information with those included in its updated routing table. Based on the Destination, Flooding $I D$ and $S N$ fields, the forwarder vehicle can find out automatically the next zone to the target destination. The same maneuver is executed by all forwarders located in the selected zones' succession until gateway $y_{5}$. When the destination node receives the data packet, it checks the Destination field in its header to confirm the reception of the data packet. In the case where the received data packet is not intended for this destination node, the current node checks its routing table to find the right next zone where the data packet will be forwarded to it.

4) Path failure: To illustrate the maintenance strategy, we give a concrete example depicted in Fig. 9 by zooming in Fig. 5 If, for example, the selected path is disconnected at the zone $I$, the closest node to the destination has to be found to continue the data transmission. The current forwarder located in the zone $H$ has to check the connectivity of the next zones other than $I$. After checking its routing table and table of neighbors, the current vehicle finds the zone $O$ as an alternative zone which is known for its closer geographical location to the destination and it can have an available path to the destination. At the same time, the vehicle has to generate a route error (RERR) packet which is sent back to the source in order to re-initiate the route discovery if there are future transmissions.

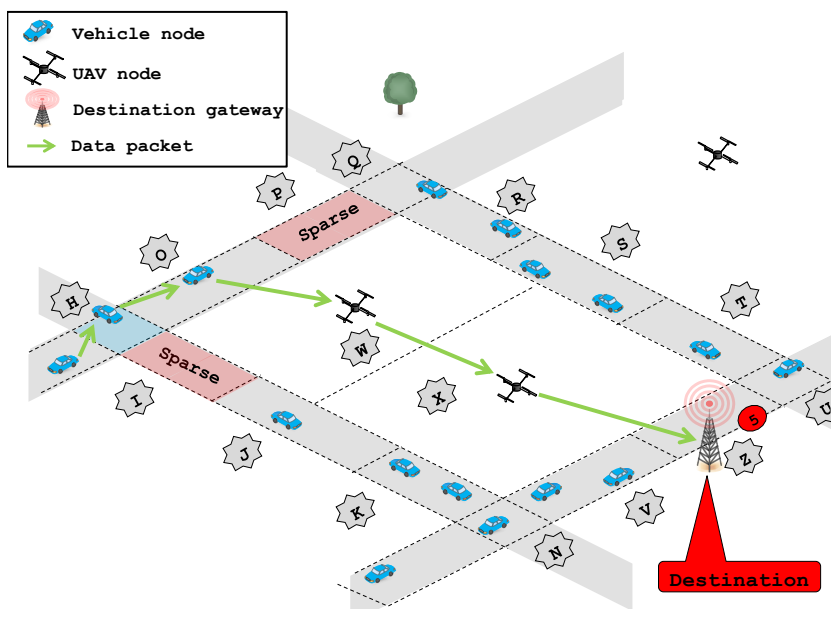

Fig. 9: Recovered path failure.

Most of the time, the alternative connected zones can be found in the sky through UAVs. Moreover, any path failure can be predicted prior to its occurrence where the maintenance strategy takes place. The mechanism used during the maintenance strategy can reduce significantly the overhead caused by the flooding of control packets. 


\section{EVALUATION}

To evaluate the performances of our protocol we use the simulation tool NS-2. The mobility of vehicles is generated by SUMO. These movements are based on the map shown in Fig. 10 For the UAVs, a Random Walk mobility is adopted for 10 UAVs in which their altitude does not exceed 300 meters during the flight. All simulations are carried out under the parameters presented in TABLE III

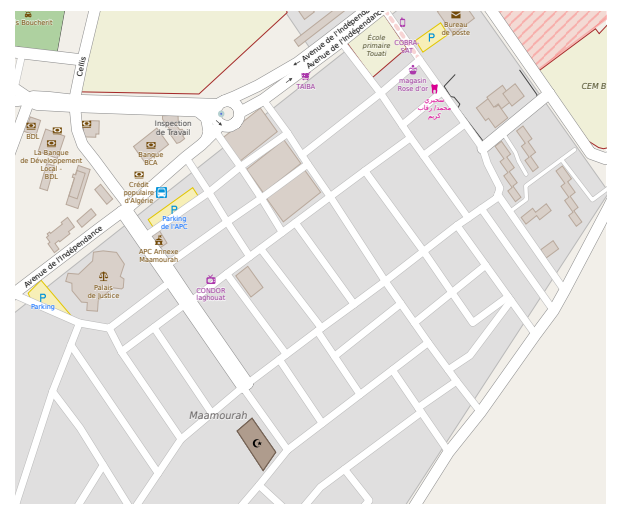

Fig. 10: Map of the simulation (N 334' $51.5^{\prime \prime}$ E $2^{\circ} 51^{\prime} 58.9^{\prime \prime}$ ).

A set of evaluation metrics is calculated during the simulation such as the packet delivery ratio (PDR), the endto-end delay (EED), the overhead $(\mathrm{OH})$, and the average number of hops (HOP). Our approach is evaluated under two different communication ranges $(R 2 \approx 300 \mathrm{~m}$ and $R 1 \approx 1000 \mathrm{~m})$ and compared with RBVT-R [24], OLSR [25], and with our previous UAV-assisted protocols CRUV [5] and UVAR [6]. We note that RBVT-R and OLSR do not consider the existing UAVs during the simulation. Fifty pairs of mobile nodes are selected randomly to establish a communication between each other. Moreover, the Hello interval is set to 0.1 (s). The list of
TABLE III: Simulation parameters

\begin{tabular}{|c|c|c|}
\hline & Parameter & Value \\
\hline \multirow{9}{*}{ 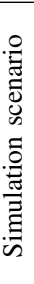 } & Area size & $4 \times 4 \mathrm{~km}^{2}$ \\
\hline & Simulation time & $900 \mathrm{~s}$ \\
\hline & Mobility generator & SUMO \\
\hline & Number of intersections & 47 \\
\hline & Number of roads & 100 \\
\hline & Number of vehicles & {$[80,320]$} \\
\hline & Number of UAVs & 10 \\
\hline & Vehicle speed & {$[0,50] \mathrm{km} / \mathrm{h}$} \\
\hline & UAV speed & {$[0,60] \mathrm{km} / \mathrm{h}$} \\
\hline \multirow{8}{*}{ 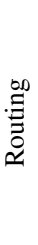 } & Frequency Band & $5.9 \mathrm{GHz}$ \\
\hline & PHY model & IEEE $802.11 \mathrm{p}$ \\
\hline & Communication range of vehicles & $\approx 300 \mathrm{~m}$ \\
\hline & Communication range of UAVs & $R 1 \approx 300 \mathrm{~m}$ and $R 2 \approx 1000 \mathrm{~m}$ \\
\hline & Data size & $1 \mathrm{~KB}$ \\
\hline & Number of packets senders & 50 \\
\hline & Channel capacity & $6 \mathrm{Mbps}$ \\
\hline & $\%$ of nodes requesting data & $20 \%$ \\
\hline
\end{tabular}

neighboring nodes and the routing table included in all nodes are purged after 10 (s) of no activity.

\section{A. Results analysis}

In Fig. 8(a) and box plots in Fig. 8(e), we clearly distinguish that our approach achieves a high PDR in both communication ranges compared with other protocols (more than $80 \%$ ). This can be justified by the used mechanism based on $P E$ in both the data delivery and the maintenance strategy. Moreover, when $R 2 \approx 1000 \mathrm{~m}$, the network becomes more connected and more data packets are successfully sent without any losses. We also notice the higher delivery ratios of RBVT-R and OLSR comparing with those of UVAR and CRUV. Firstly, because RBVT-R is a reactive-based routing protocol working without using a penalizing technique such as the carry-and-forward which is employed by UVAR and CRUV. Secondly, because in OLSR, all routes are computed beforehand and data packets can be quickly forwarded or dropped at intermediate nodes.

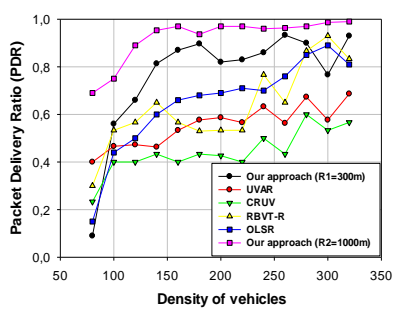

(a) PDR.

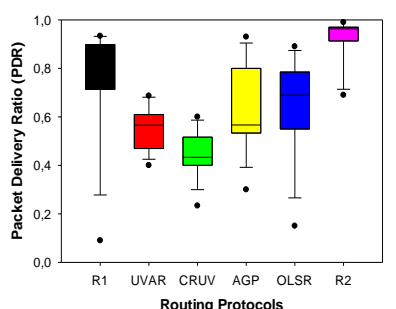

(e) Average PDR.

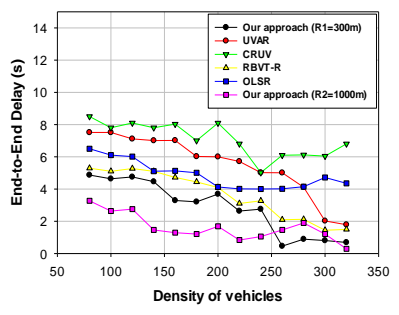

(b) EED.

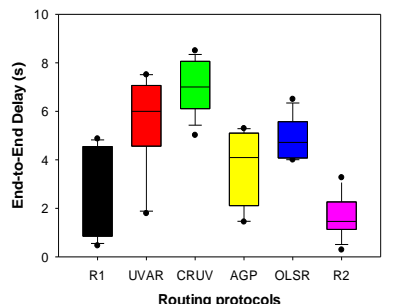

(f) Average EED.

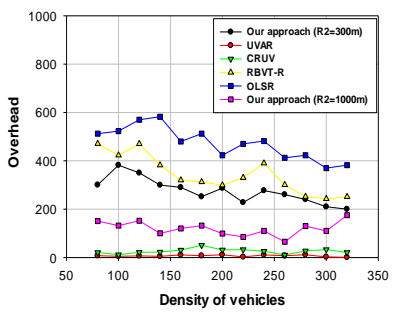

(c) $\mathrm{OH}$.

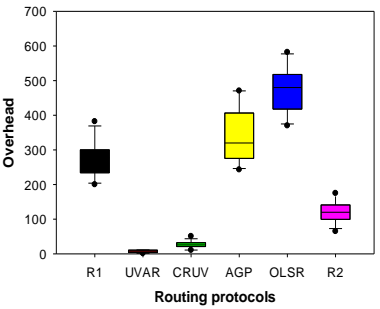

(g) Average $\mathrm{OH}$.

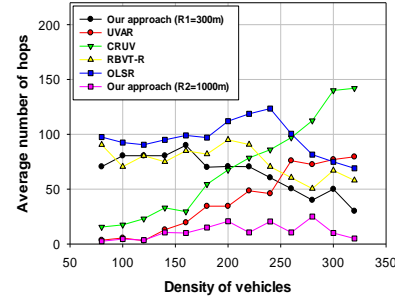

(d) HOP.

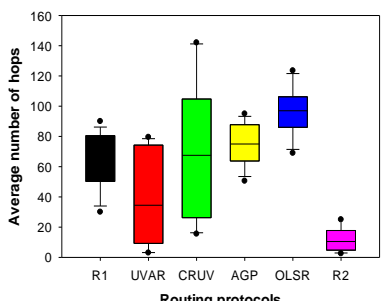

(h) Average HOP.

Fig. 8: Simulation results vs. Density of vehicles $(U A V s=10)$. 
Fig. 8(b) and the box plots in Fig. 8(f) clearly show that our approach achieves the lowest delay for all vehicle densities in both ranges. This is explained by the low durations taken during the route discovery and the calculated $P E$, which allow to avoid the re-initialization of the route discovery at each path failure. However, the other protocols do not perform as well as our approach. Thus, it can be explained by the permanent reinitialization of the route discovery or inadequate mechanisms used during the data delivery.

Figs. $8(\mathrm{c})$ and $8(\mathrm{~g})$ show that the control overhead of the reactive protocols (Our approach in both ranges and RBVT-R) and the proactive protocol OLSR is important compared with CRUV or UVAR. This is mainly due to the generated control packets during the route discovery and the periodical exchange of link states between the nodes, respectively. However, as a reactive protocol, our approach has the lowest overhead where small overhead is generated thanks to the used maintenance mechanism and the static size of the control packets.

Figs. 8(d) and 8(h) show that initially (low densities 80$180)$, our approach $(R 1 \approx 300 m)$ has an important number of hops compared with UVAR and CRUV, which is essentially due to the permanent re-initialization of the route discovery due to disconnections. However, in the case when $R 2 \approx$ $1000 \mathrm{~m}$, the number of hops is decreased due to the use of the greedy forwarding technique, and particularly, through UAVs that provide a large coverage.

\section{CONCLUSION}

In this paper, a novel UAV-assisted reactive routing protocol has been introduced. This protocol exploits in conjunction with the flooding process, a predictive technique to estimate accurately the expiration time of the discovered routing paths. Despite their small coverage and low altitudes, UAVs have demonstrated their effectiveness to be the crucial support as relays during the routing and as multiplication points of several alternative solutions during the maintenance strategy in the case of path failures. Performance results clearly justify our claims in terms of delivery ratio and delay of transmission for the use of a combined UAV-VANET network. Given such performances, another part of this work which we are currently investigating is the control of the UAV mobility to detect path failures and place UAVs as a bridge between two disconnected parts of the network. Moreover, another study, which can be further taken into consideration as future perspectives is the incorporation of a security component, including misbehavior detection systems, digital signatures, and other cryptography schemes in order to deal with some kinds of attacks.

\section{REFERENCES}

[1] O. S. Oubbati, A. Lakas, F. Zhou, M. Güneş, N. Lagraa, and M. B. Yagoubi, "Intelligent UAV-Assisted Routing Protocol for Urban VANETs," Computer communications, vol. 107, pp. 93-111, 2017.

[2] S. Bitam, A. Mellouk, and S. Zeadally, "VANET-cloud: a generic cloud computing model for vehicular ad hoc networks," IEEE Wireless Communications, vol. 22, no. 1, pp. 96-102, 2015.

[3] O. S. Oubbati, A. Lakas, F. Zhou, M. Güneş, and M. B. Yagoubi, "A survey on position-based routing protocols for Flying Ad hoc Networks (FANETs)," Vehicular Communications, vol. 10, pp. 29-56, 2017.
[4] Y. Zhou, N. Cheng, N. Lu, and X. S. Shen, "Multi-UAV-aided networks: Aerial-ground cooperative vehicular networking architecture," IEEE Vehicular Technology Magazine, vol. 10, no. 4, pp. 36-44, 2015.

[5] O. S. Oubbati, A. Lakas, N. Lagraa, and M. B. Yagoubi, "CRUV: Connectivity-based traffic density aware routing using UAVs for VANETs," in Proceedings of International Conference on Connected Vehicles and Expo (ICCVE), 2015, pp. 68-73.

[6] O. S. Oubbati, A. Lakas, N. Lagraa, and M. B. Yagoubi, "UVAR: An intersection uav-assisted vanet routing protocol," in Wireless Communications and Networking Conference (WCNC), 2016 IEEE. IEEE, 2016, pp. $1-6$.

[7] O. S. Oubbati, A. Lakas, M. Güneş, F. Zhou, and M. B. Yagoubi, "UAVassisted reactive routing for urban VANETs," in Symposium on Applied Computing. ACM, 2017, pp. 651-653.

[8] T. Taleb, E. Sakhaee, A. Jamalipour, K. Hashimoto, N. Kato, and Y. Nemoto, "A stable routing protocol to support ITS services in VANET networks," IEEE Transactions on Vehicular Technology,, vol. 56, no. 6, pp. 3337-3347, 2007.

[9] P. Li, T. Miyazaki, K. Wang, S. Guo, and W. Zhuang, "Vehicle-assist resilient information and network system for disaster management," IEEE Transactions on Emerging Topics in Computing, vol. 5, no. 3, pp. 438-448, 2017.

[10] J. Li, G. Deng, C. Luo, Q. Lin, Q. Yan, and Z. Ming, "A hybrid path planning method in unmanned air/ground vehicle (UAV/UGV) cooperative systems," IEEE Transactions on Vehicular Technology, vol. 65, no. 12, pp. 9585-9596, 2016.

[11] Z. M. Fadlullah, D. Takaishi, H. Nishiyama, N. Kato, and R. Miura, "A dynamic trajectory control algorithm for improving the communication throughput and delay in UAV-aided networks," IEEE Network, vol. 30, no. 1, pp. 100-105, 2016.

[12] L. Xiao, X. Lu, D. Xu, Y. Tang, L. Wang, and W. Zhuang, "UAV relay in VANETs against smart jamming with reinforcement learning," IEEE Transactions on Vehicular Technology, vol. 67, no. 5, pp. 4087-4097, 2018.

[13] R. Shirani, M. St-Hilaire, T. Kunz, Y. Zhou, J. Li, and L. Lamont, "On the delay of reactive-greedy-reactive routing in unmanned aeronautical ad-hoc networks," Procedia Computer Science, vol. 10, pp. 535-542, 2012.

[14] M. Iordanakis, D. Yannis, K. Karras, G. Bogdos, G. Dilintas, M. Amirfeiz, G. Colangelo, and S. Baiotti, "Ad-hoc routing protocol for aeronautical mobile ad-hoc networks," in Proceeding of the Fifth International Symposium on Communication Systems, Networks and Digital Signal Processing (CSNDSP). Citeseer, 2006.

[15] S. D. Muruganathan, X. Lin, H.-L. Maattanen, Z. Zou, W. A. Hapsari, and S. Yasukawa, "An Overview of 3GPP Release-15 Study on Enhanced LTE Support for Connected Drones," arXiv preprint arXiv:1805.00826, 2018.

[16] L. Gupta, R. Jain, and G. Vaszkun, "Survey of important issues in UAV communication networks," IEEE Communications Surveys \& Tutorials, vol. 18, no. 2, pp. 1123-1152, 2016.

[17] K. Dalamagkidis, "Classification of UAVs," in Handbook of Unmanned Aerial Vehicles. Springer, 2015, pp. 83-91.

[18] C. Sommer, D. Eckhoff, R. German, and F. Dressler, "A computationally inexpensive empirical model of ieee $802.11 \mathrm{p}$ radio shadowing in urban environments," in Proceedings of the Eighth IEEE International Conference on Wireless On-Demand Network Systems and Services (WONS), 2011, pp. 84-90.

[19] J. Li and Y. Han, "Optimal resource allocation for packet delay minimization in multi-layer UAV networks," IEEE Communications Letters, vol. 21, no. 3, pp. 580-583, 2017.

[20] Q. W. . R. Zhang, "Common Throughput Maximization in UAV-Enabled OFDMA Systems With Delay Consideration," IEEE Transactions on Communications, vol. 66, no. 12, pp. 6614- 6627, 2018.

[21] W. Su, S.-J. Lee, and M. Gerla, "Mobility prediction and routing in ad hoc wireless networks," International Journal of Network Management, vol. 11, no. 1, pp. 3-30, 2001.

[22] M. Uddin et al., "Link expiration time-aware routing protocol for UWSNs," Journal of Sensors, vol. 2013, pp. 1-9, 2013.

[23] K. Donald et al., "The art of computer programming, volume 2: Semi numerical algorithms," 1998.

[24] J. Nzouonta, N. Rajgure, G. Wang, and C. Borcea, "VANET routing on city roads using real-time vehicular traffic information," IEEE Transactions on Vehicular Technology, vol. 58, no. 7, pp. 3609-3626, 2009.

[25] T. Clausen and P. Jacquet, "Optimized link state routing protocol (OLSR),” Tech. Rep., 2003. 